\title{
Associação entre doenças crônicas em adultos e redução dos niveis de atividade física
}

\author{
Chronic diseases in adults promotes reduction of the level \\ of physical activity
}

Bruna Camilo Turi', Jamile Sanches Codogno², Rômulo Araújo Fernandes ${ }^{3}$, Henrique Luiz Monteiro ${ }^{4}$

\begin{abstract}
RESUMO
Modelo do estudo: Estudo de prevalência. Objetivo: Descrever a ocorrência das doenças mais frequentes que acometem portadores de hipertensão arterial (HAS) e/ou diabetes mellitus (DM), usuários de Unidade Básica de Saúde (UBS) e associar a ocorrência das duas doenças com o nível de atividade física. Métodos: Estudo transversal envolvendo 222 indivíduos portadores de hipertensão arterial e/ou diabetes mellitus, de ambos os sexos e usuários de duas UBS da cidade de Bauru/SP. Foram analisados prontuários clínicos dos pacientes para verificação e constatação da ocorrência de doenças. Peso corporal e estatura foram aferidos para classificação do estado nutricional e foi aplicado questionário para verificar o nível de atividade física. O teste qui-quadrado foi utilizado para analisar existência de associações e a regressão logística binária expressou a magnitude das mesmas. As comparações foram consideradas significativas quando $p<0,05$. Resultados: A amostra teve predominância de mulheres $(64 \%)$, pessoas com obesidade $(42,3 \%)$ e sedentarismo $(78,8 \%)$. As doenças de maior ocorrência entre os pacientes hipertensos e diabéticos foram a hipercolesterolemia (31,5\%), seguida pela artrite/artrose (23,8\%) e lombalgia (23.8\%). Houve associação significativa entre indicadores de prática de atividades físicas no lazer (intensidade, tempo semanal de prática e engajamento prévio) com menor ocorrência de doenças metabólicas. Independente de variáveis de confusão, indivíduos diabéticos e diabéticos com hipertensão tiveram chances aumentados de apresentar comportamento sedentário. Conclusão: Os resultados sugerem que o acometimento de pacientes hipertensos e/ou diabéticos por comorbidades está associado às altas taxas de inatividade física e, conseqüentemente, ao aumento do número de agravos associados.
\end{abstract}

Palavras-chave: Atividade física, diabetes mellitus, hipertensão arterial

\section{Introdução}

Ao longo dos últimos anos, observa-se um aglomerado de alterações no estilo de vida e em caracte- rísticas epidemiológicas que têm favorecido o desenvolvimento de doenças crônicas, como hipertensão arterial sistêmica (HAS) e diabetes mellitus tipo 2 (DM2). ${ }^{1}$
1 Mestrando. Programa de Pós-Graduação em Fisioterapia. Faculdade de Ciências e Tecnologia, UNESP - Presidente Prudente

2 Doutorando, bolsista CAPES. Programa de Pós-Graduação em Ciências da Motricidade. Instituto de Biociências, UNESP - Rio Claro - Bolsista Capes

3 Professor Doutor. Universidade do Oeste Paulista, UNOESTE Presidente Prudente

4 Departamento de Educação Física. Faculdade de Ciências, UNESP - Bauru
Universidade Estadual Paulista. Faculdade de Ciências. Departamento de Educação Física. Av. Eng. Luiz Edmundo Carrijo Coube, 14-01 . CEP: 17033-360 - Bauru/SP E-mail: brunaturi@ hotmail.com

Artigo recebido em 07/06/2011 Aprovado para publicação em 14/12/2011 
Em todo o mundo, estima-se que estes desfechos patológicos acometam 972 milhões de hipertensos e cerca de 194 milhões de diabéticos e as projeções indicam que haverá aumento no número de pessoas portadoras desses agravos. ${ }^{2,3}$ No Brasil o quadro não é diferente, estudos encontraram prevalências elevadas para HAS e DM. ${ }^{4,5}$

Para controle, prevenção e/ou tratamento de tais patologias, há evidências de que a prática continuada de atividades físicas ao longo da vida configura-se como hábito importante, contribuindo para, entre outros benefícios, manter o perfil glicêmico, lipídico e a pressão arterial dentro de limites desejáveis ${ }^{6,7,8}$, ficando o indivíduo que mantém um estilo de vida sedentário sujeito a ser acometido por inúmeras doenças crônicas. No presente estudo se propõe um novo olhar sobre o problema, na medida em que o portador dessas patologias pode vir a apresentar o comportamento sedentário, sendo agora a exposição do indivíduo determinada pela HAS e DM2 e seu desfecho o baixo nível de atividade física.

Acerca de tais considerações, vislumbra-se como relevante aprofundar o conhecimento sobre a interação entre ocorrência de doenças e prática de atividades físicas da população usuária do serviço público de atenção primária à saúde, uma vez que essas informações poderão nortear a adoção de ações que proporcionem o acesso à prática de atividades físicas, e desse modo prevenir os agravos que podem ser causados pelo sedentarismo. ${ }^{9}$

Assim, os objetivos do presente estudo foram: I) descrever a ocorrência das doenças mais frequentes que acometem portadores de hipertensão arterial e/ou diabetes mellitus, usuários de Unidade Básica de Saúde (UBS), e II) associar a ocorrência das duas doenças com o nível de atividade física.

\section{Materials e métodos}

\section{Amostra}

A cidade de Bauru tem 17 Unidades Básicas de Saúde (UBS) que recebem recursos do Sistema Único da Saúde (SUS). Após contato com a Secretaria Municipal de Saúde, foi sugerida a realização do estudo em duas destas UBS, particularmente pelas características semelhantes dos bairros e perfil sócio econômico dos usuários. Dessa forma, a casuística do presente estudo foi formada por pacientes cadastrados nas UBS "Octávio Rasi" e "Vila Cardia". Pre- viamente a realização da pesquisa, foram definidos os seguintes critérios de inclusão: (I) apresentar hipertensão arterial e/ou diabetes mellitus tipo 2; (II) ter, no mínimo, 30 anos de idade; (III) ter prontuário ativo na UBS há um ano; (IV) ser o único serviço ambulatorial com que mantém acompanhamento regular de saúde. Nas duas UBS, todos os pacientes que atendiam os critérios de inclusão foram previamente agendados pela enfermeira responsável e convocados para o dia da consulta. Todos os que responderam à convocação foram avaliados, resultando em um grupo composto por 222 pessoas, sendo 80 homens e 142 mulheres.

O projeto foi aprovado pelo Comitê de Ética em Pesquisa (Protocolo 6898-2008) e todos os pacientes assinaram o Termo de Consentimento Livre e Esclarecido.

\section{Avaliações}

Os entrevistadores e avaliadores foram previamente treinados pelo coordenador da pesquisa. As variáveis: sexo, idade, peso corporal, estatura e índice de massa corporal (IMC) foram utilizadas para definir o perfil da população estudada. O IMC $\left(\mathrm{kg} / \mathrm{m}^{2}\right)$ foi calculado com a utilização dos valores de massa corporal (balança digital com precisão de $0,1 \mathrm{~kg}$ e capacidade máxima de $150 \mathrm{~kg}$ ) e estatura (estadiômetro com precisão de $0,1 \mathrm{~cm}$ e extensão máxima de 2 metros), que foram coletados segundo o protocolo de Lohman et al. ${ }^{10}$. Os valores de corte para sobrepeso $\left(25-29,9 \mathrm{~kg} / \mathrm{m}^{2}\right)$ e obesidade $\left(\geq 30 \mathrm{~kg} / \mathrm{m}^{2}\right)$ foram adotados de acordo com classificação proposta pela Organização Mundial da Saúde (OMS). ${ }^{11}$

\section{Prática de atividades físicas}

A prática atual de atividades físicas durante horário de lazer foi avaliada através do questionário de Baecke et al. ${ }^{12}$ (sessão-2 do instrumento [atividades esportivas]). Foram analisados três construtos dessa prática de atividades físicas durante horários de lazer: intensidade (baixa, média e alta), tempo semanal de prática (<1h/sem; 1-2h/sem; 2-3h/sem; 3-4h/ sem; $>4 \mathrm{~h} / \mathrm{sem})$ e tempo prévio de engajamento $(<1$ mês; 1-3 meses; 4-6 meses; 7-9 meses; $>9$ meses). Assim, foram considerados fisicamente ativos os indivíduos que reportaram um mínimo de 180 minutos por semana (3-4h/sem) de atividades físicas de intensidade moderada ou vigorosa nos últimos quatro-seis meses. Por fim, seguindo metodologia prévia ${ }^{6,8}$, criou-se uma variável que classificou os indivíduos segundo três 
categorias, a saber: (i) Sedentários: aqueles que não referiram prática alguma de atividades físicas; (ii) Insuficientemente Ativos: os que realizaram menos do que os 180 minutos por semana / ou intensidade abaixo da estabelecida / ou menor tempo prévio de engajamento; e, (iii) Suficientemente Ativos: os que alcançaram o ponto de corte proposto.

\section{Ocorrência de doenças}

A ocorrência de doenças foi obtida por meio de informações auto-referidas pelos pacientes (hipertensão arterial sistêmica [HAS], hipercolesterolemia, diabetes mellitus tipo 1 e 2 , hipertiroidismo, hipotiroidismo, acidente vascular cerebral [AVC], arritmia, infarto, angina, doenças tumorais, osteoporose, lombalgia, artrite/artrose, escoliose, bronquite, labirintite, apendicite, bursite, depressão e gota). Os dados informados pelos indivíduos foram confrontados com os registros contidos nos prontuários clínicos, retroagindo 12 meses à data da entrevista, buscando investigar se, no auto-referimento, houve omissão ou esquecimento de doenças (em caso de divergência, o registro médico no prontuário foi adotado como correto). De acordo com a Classificação Estatística Internacional de Doenças e Problemas Relacionados à Saúde (CID-10) versão $2008^{13}$, estas doenças foram agrupadas em: cardiovasculares, metabólicas, osteoarticulares e outras. Adicionalmente, criou-se uma variável de acordo com o número de agravos que acometiam a amostra.

\section{Análise Estatística}

As variáveis analisadas foram expressas sob a forma de distribuição de frequências absolutas e relativas (\%). O teste qui-quadrado analisou possíveis associações e a regressão logística binária (expressa em valores de odds ratio [OR] e intervalos de confiança de 95\% [IC95\%]) expressou a magnitude das mesmas. As comparações foram consideradas significativas quando a significância estatística obtida foi inferior a 5\%. Foi utilizado o software estatístico Statistical Package for the Social Sciences (SPSS), versão 13.0.

\section{Resultados}

Entre os pacientes avaliados houve predominância de mulheres $(n=142$ [64\%]) e pessoas com obesidade $(n=94[42,3 \%])$. A média de idade foi de $60,7 \pm$ 9,3 anos. A faixa etária que concentrou maior número de indivíduos foi a de 50-64,9 anos $(61,7 \%)$, seguida daqueles com $>65$ anos $(28,4 \%)$ e de 30 - 49,9 anos $(9,9 \%)$. Sedentários (nenhuma prática de atividade física no lazer) somaram 78,8\% dos avaliados, $16,7 \%$ foram classificados como insuficientemente ativos e os suficientes ativos representaram 4,5\% ([IC95\%= $2,3 \%-8,2 \%])$ dos sujeitos investigados $(\mathrm{n}=10)$.

Considerando a casuística como um todo: $26,1 \%$ eram apenas hipertensos, $14 \%$ eram apenas diabéticos e 59,9\% apresentavam ambos os desfechos. Quando analisadas as doenças com maior ocorrência nos hipertensos e diabéticos, as taxas mais elevadas foram: hipercolesterolemia $(31,5 \%)$, artrite/artrose $(23,8 \%)$ e lombalgia $(23,8 \%)$ (Figura 1$)$ e as de menor ocorrência foram: depressão, labirintite e hipotireoidismo (1\% para todas). O percentual de doenças osteoarticulares foi de $33,8 \%$ e outras doenças foi $27,5 \%$.

A Tabela 1 indica que, quando analisados separadamente os indicadores da prática de atividades físicas no lazer (intensidade, tempo semanal de prática e engajamento prévio), para todos os indicadores, houve relação significativa com menor ocorrência de doenças metabólicas.

Independente do efeito de possíveis variáveis de confusão (Figura 2, Painéis A e B), quando comparados com indivíduos hipertensos, aqueles com diabetes mellitus tipo $2(\mathrm{OR}=3.69$ [1.21-9.20]) e com ambas as doenças $(\mathrm{OR}=5.12$ [2.45-9.60]) apresentaram chances aumentadas de serem classificados como sedentários.

\section{Discussão}

Dos pacientes avaliados, considerando a prática de exercícios físicos no lazer, constatamos alta taxa de sedentarismo $(78,8 \%)$. No entanto, este resultado é convergente com as faixas encontradas em revisão sistemática de estudos brasileiros sobre inatividade física, que variaram entre 55,3\% e 96,7\%. ${ }^{14}$ Quando analisadas amostras compostas por adultos não necessariamente acometidos por alguma doença crônica, os valores obtidos foram superiores aos observados em São Paulo-SP $(65,4 \%)$ e Goiânia-GO $(61,9 \%) .{ }^{15,16}$ Assim, destaca-se a enorme ocorrência de sedentarismo nos indivíduos atendidos pela rede pública de saúde, salientando que estratégias de promoção da prática de atividades físicas fazem-se necessárias nesta população.

Assim como o sedentarismo, a obesidade também é considerada fator de risco para doenças crônicas ${ }^{17}$ e pode contribuir para a redução dos níveis de 


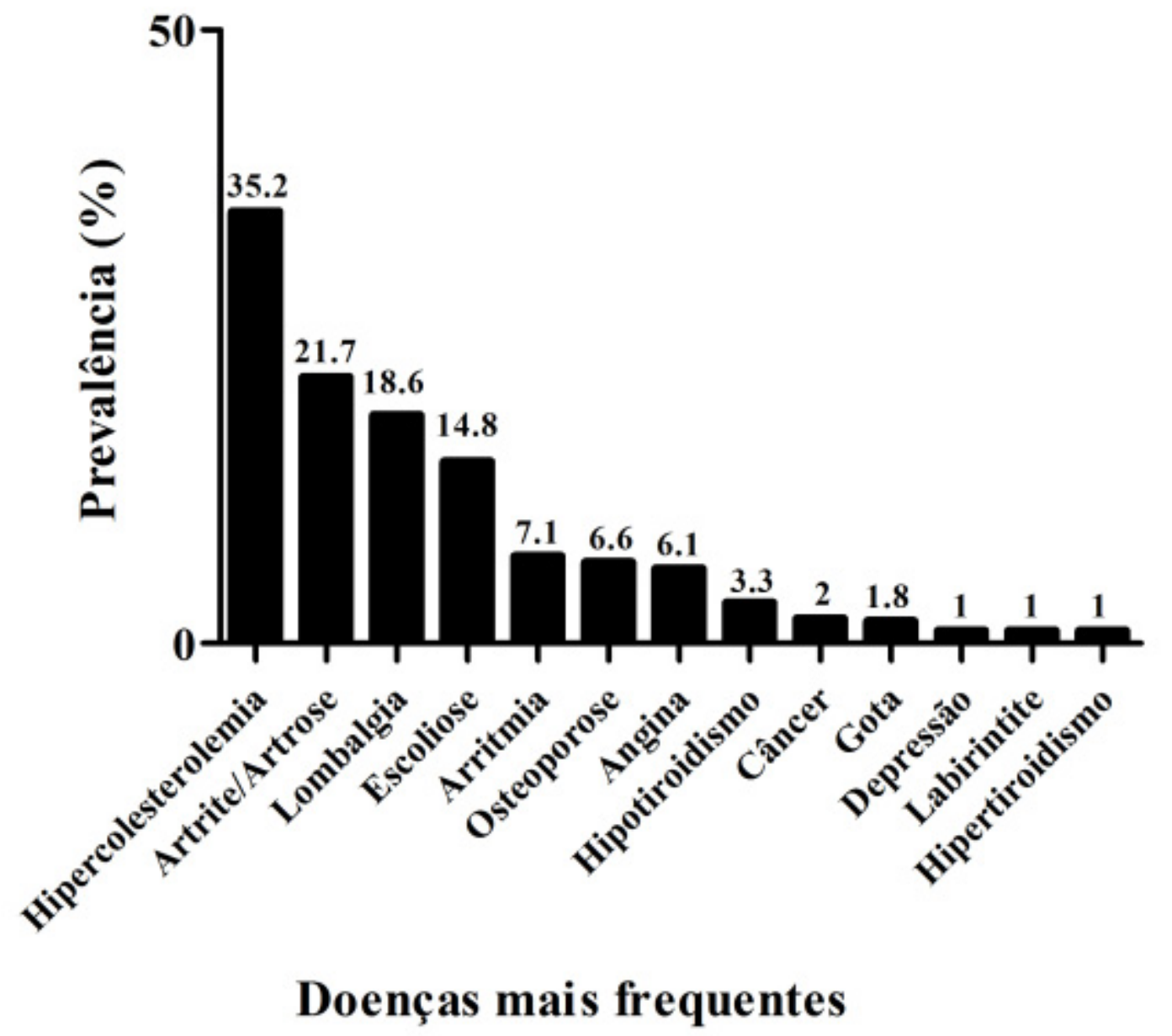

Figura 1. Ocorrência de doenças em usuários do Sistema Único de Saúde com hipertensão ou diabetes mellitus tipo 2 (Bauru, 2009).

\section{Tabela 1}

Correlação de Spearman entre grupos de doença e parâmetros da atividade física realizada no lazer

Grupos de doença de acordo com CID-10

Metabólicas

Atividade Física

(E00-E90)
Cardiovasculares

(I00-I99)
Osteoarticulares

(M00-M99) Outras

\begin{tabular}{lcccc} 
& $\mathrm{r}=$ & $\mathrm{r}=$ & $\mathrm{r}=$ & $\mathrm{r}=$ \\
\hline Intensidade & $-0.304^{*}$ & 0.003 & -0.038 & -0.047 \\
Horas por semana & $-0.326^{*}$ & -0.002 & -0.044 & -0.050 \\
Tempo de engajamento & $-0.317^{*}$ & 0.006 & -0.024 & -0.045 \\
\hline
\end{tabular}

$*=\mathrm{p}<0,01$ 


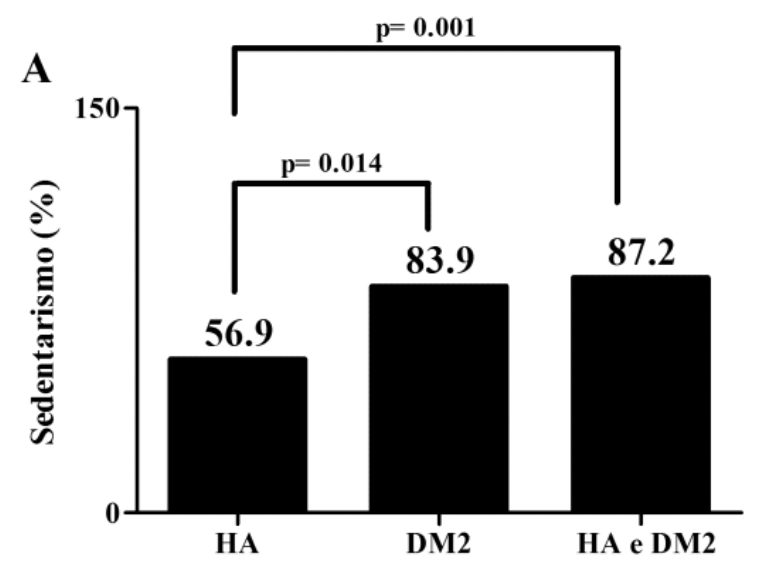

\section{B}

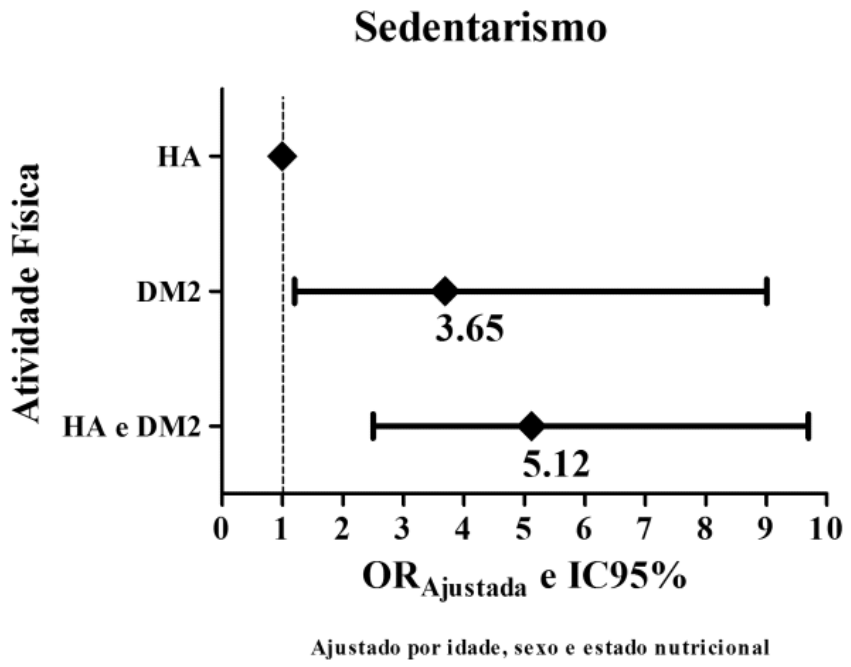

Figura 2. Associação entre a presença de hipertensão arterial (HA) e diabetes mellitus tipo 2 (DM2) e sedentarismo em usuários do Sistema Único de Saúde (Bauru, 2009).

atividade física do indivíduo acima do peso. Em nossa amostra, 42,3\% dos pacientes foram classificados como obesos, resultado semelhante aos de Cipullo et al. ${ }^{18}$, que encontraram 55,4\% dos indivíduos estudados com excesso de peso ou obesidade, além de encontrarem maior prevalência de HAS para indivíduos com sobrepeso ou obesos quando comparados àqueles com IMC normal.

Da mesma maneira, estudo transversal de base populacional encontrou prevalências de excesso de peso em adultos variando de $49,3 \%$ a $61,2 \%$ dependendo da faixa de idade, e observou que $64,9 \%$ dos hipertensos estavam com peso acima do limite aceitável. ${ }^{19}$

Similarmente, Banks et al. ${ }^{20}$, em estudo que buscou examinar a relação entre obesidade, atividades físicas domésticas e sedentarismo na Tailândia, encontraram $15,6 \%$ de indivíduos obesos na amostra (similarmente ao nosso achado) e observaram associações inversas entre ser obeso e o total de sessões semanais de atividades físicas. Além disso, houve aumento da obesidade com o aumento do tempo em frente a televisão, com incremento de $18 \%$ a cada duas horas diárias de tempo adicional. Também observaram $33 \%$ menos risco de ser obeso entre aqueles que faziam trabalho doméstico ou jardinagem diariamente quando comparados aos que nunca faziam essas atividades.
Em nossa investigação foi observada associação entre elevadas taxas de inatividade física e acometimento dos pacientes hipertensos e/ou diabéticos por doenças osteoarticulares, como artrite/artrose e lombalgia. Esse comportamento sedentário pode ser explicado justamente pelo fato de os indivíduos acometidos pela associação dessas morbidades ficarem impossibilitados de praticar atividades físicas ao se depararem com sintomas de dor, que pioram com a sobrecarga que surge no início da marcha, e se intensificam no fim do dia ${ }^{21}$. Adicionalmente, vale destacar a possível presença da neuropatia, que pode elevar as chances de pacientes diabéticos desenvolverem úlceras nos membros inferiores ${ }^{22} \mathrm{e}$, dessa forma, ajudaria a justificar a elevada ocorrência de agravos osteoarticulares.

Entre os adultos hipertensos e/ou diabéticos usuários de UBS avaliados foram encontradas relações entre ocorrência de comorbidades e diferentes indicadores do nível de atividade física no lazer (intensidade, tempo semanal de prática e engajamento prévio), indicando que mesmo diante da presença de ambos os desfechos, pode haver associação significativa entre maior prática de atividades físicas e menor ocorrência de outras doenças metabólicas. Corroborando, trabalhos recentemente publicados indicaram significante associação entre maior prática de atividades físicas no lazer (180 minutos de atividades de intensidade moderada ou elevada por mais de 4 
meses) e menor ocorrência de doenças metabólicas entre adultos ${ }^{6,8}$. Adicionalmente, os autores identificaram que tal associação foi mediada pelo efeito da atividade física na composição corporal.

No que se refere à maior inatividade física entre indivíduos diabéticos e com hipertensão arterial, algumas hipóteses podem ser formuladas com base na literatura. Huang (2009) ${ }^{23}$ postula que uma das justificativas para a grande ocorrência de hipertensão arterial entre diabéticos é baseada na disfunção endotelial ocasionada pela resistência à ação da insulina. Nesse sentido, existem evidências do efeito benéfico da prática de exercícios físicos sobre o tecido endotelial ${ }^{24}$ e captação de glicose dos tecidos, sendo que este efeito ocorre independentemente da ação da insulina, por meio da ativação da enzima $\mathrm{AMPK}^{25}$.

Com as evidências encontradas, dá-se ênfase à importância de programas que promovam cuidados à saúde, como prática de atividade física supervisionada, programas de intervenção em exercícios específicos e orientação nutricional, a fim de prevenir o aparecimento e/ou diminuir a prevalência de outras doenças crônicas que acometem essa população.

As principais limitações do presente estudo merecem ser destacas. Inicialmente, o auto-relato das doenças pode constituir uma limitação, pois, pode subestimar de maneira significativa a ocorrência das mesmas. Porém, os autores acreditam que a utiliza- ção do prontuário médico como uma "medida de controle" tenha atenuado de maneira sensível tal limitação. A segunda limitação refere-se ao delineamento transversal do estudo, o qual não permite estabelecer relações de causalidade entre o desfecho e as variáveis independentes. Assim, não é possível determinar com total certeza que os pacientes tornaram-se diabéticos ou hipertensos em decorrência do estilo de vida sedentário, ou tornaram sedentários após serem acometidos pelas doenças. Fernandes et al. ${ }^{26}$ apontam que em estudos epidemiológicos nos quais não é viável utilizar delineamentos prospectivos, a utilização de medidas retrospectivas de atividade física pode ser uma saída interessante ao modelo convencional de medidas transversais. Dessa forma, sugere-se que futuros estudos abordando a temática sejam realizados na tentativa de suplantar tais limitações e avançar no entendimento sobre o tema.

\section{Conclusão}

Em resumo, conclui-se que as doenças mais prevalentes entre os pacientes avaliados foram a hipercolesterolemia, seguida pela artrite/artrose e lombalgia. Além disso, os resultados sugerem que o acometimento de pacientes hipertensos e/ou diabéticos por comorbidades está associado às altas taxas de inatividade física.

\section{ABSTRACT}

Type of study: Prevalence study. Objective: To describe the occurrence of diseases in hypertensive and/ or diabetic patients, users of Basic Health Unit (BHU) and to associate the occurrence of both diseases with physical activity. Methods: Cross-sectional study involving 222 patients with hypertension and/or diabetes mellitus, of both sexes and users of two BHU from Bauru, Brazil. Clinical records of the patients were analyzed to detect the presence of diseases, body weight and height were measured to determine nutritional status, as well as, a questionnaire assessed the physical activity level. Chi-square test identified associations and the binary logistic regression expressed the magnitude of these associations. Significant was set at $p<0.05$. Results: The sample had more women $(64 \%)$, obese $(42.3 \%)$ and sedentary persons (78.8\%). The most frequent diseases among the patients were hypercholesterolemia $(31.5 \%)$, arthritis / arthrosis (23.8\%) and low back pain (23.8\%). There was significant association between leisure physical activity (intensity, weekly practice and prior engagement) and lower occurrence of metabolic diseases. Independent of confounders, diabetic and diabetics with hypertension had increased likelihood to be sedentary. Conclusion: The results suggest that the onset of hypertension and / or diabetic co-morbidity is associated with high rates of physical inactivity and, consequently, an increased number of co-morbidities.

Key words: Physical activity, diabetes mellitus, arterial hypertension 


\section{Referências}

1. Toscano CM. As campanhas nacionais para detecção das doenças crônicas não-transmissíveis: diabetes e hipertensão arterial. Ciênc. saúde colet 2004; 9; 885-895.

2. Kearney PM, Whelton M, Reynolds K, Muntner P, Whelton P, $\mathrm{He} \mathrm{J}$. Global burden of hypertension: analysis of worldwide data. Lancet 2005; 365:217-223.

3. Federação Internacional de Diabetes. Atlas do diabetes, 2003. Disponível em www.idf.org/e-atlas

4. Passos VMA, Assis TD, Barreto SM. Hipertensão arterial no Brasil: estimativa de prevalência a partir de estudos de base populacional. Epidemiol. Serv. Saúde, 2005; 15:35-45.

5. Brasil. Indicadores de morbidade e fatores de risco. Ministério da Saúde, 2004. Disponível em http://w3.datasus.gov.br/ datasus/datasus.php

6. Fernandes RA, Zanesco A. Early physical activity promotes lower prevalence of chronic diseases in adults. Hypertens Res 2010;33:926-31.

7. Donnelly JE, Blair SN, Jakicic JM, Manore MM, Rankin JW, Smith BK; American College of Sports Medicine. American College of Sports Medicine Position Stand. Appropriate physical activity intervention strategies for weight loss and prevention of weight regain for adults. Med Sci Sports Exerc 2009; 41:459-71.

8. Fernandes RA, Sponton CHG, Zanesco A. Atividade física na infância e adolescência promove efeitos benéficos na saúde de adultos. Rev SOCERJ 2009; 22:365-72.

9. Tavares DMS. Condições de saúde de idosos diabéticos do município de Uberaba/MG. Texto Contexto Enferm, 2008; 7:342-9.

10. Dumith SC. Atividade física no Brasil: uma revisão sistemática. Cad Saúde Pública 2009; 25:415s-426s.

11. Lohman TG. Anthropometric Standardization Reference Manual. Champaign. IL, Human Kinetics Books, 1988.

12. Organização Mundial da Saúde. Obesity, Preventing and Managing the Global Epidemic: Report of the WHO Consultation on Obesity. World Health Organization: Geneva, 1998. Disponível em http://www.who.int/nutrition/publications/ obesity_executive_summary.pdf

13. Classificação Estatística Internacional de Doenças e Problemas Relacionados à Saúde (CID-10), versão 2008.

14. Baecke JA, Burema J, Frijters JE. A short questionnaire for the measurement of habitual physical activity in epidemiological studies. Am J Clin Nutr 1982; 36: 936-42.
15. Hallal PC, Dumith Sde C, Bastos JP, Reichert FF, Siqueira FV, Azevedo MR. Evolution of the epidemiological research on physical activity in Brazil: a systematic review. Rev Saude Publica. 2007; 41:453-60.

16. Zanchetta LM, Barros MBA, César CLG, Carandina L, Goldbaum M, Alves MCGP. Inatividade física e fatores associados em adultos, São Paulo, Brasil. Rev. Bras. Epidemiol., 2010, 13:387-99.

17. Malta $D$, et al. Fatores de risco e proteção para doenças crônicas não transmissíveis entre beneficiários da saúde suplementar: resultados do inquérito telefônico Vigitel, Brasil, 2008. Ciênc. saúde coletiva [online] 2011; 16 (3): 2011 22

18. Cipullo JP, Martin JFV, Ciorlia LAS, Godoy MRP, Cação JC, Loureiro AAC, et al. Prevalência e fatores de risco para hipertensão em uma população urbana brasileira. Arq Bras Cardio 2010; [online]

19. Lino MZR, Muniz PT, Siqueira KS. Prevalência e fatores associados ao excesso de peso em adultos: inquérito populacional em Rio Branco, Acre, Brasil, 2007-2008. Cad. Saúde Pública 2011;27(4):797-810.

20. Banks E, Lynette L, Seubsman S, Bain C, Sleigh A. Relationship of obesity to physical activity, domestic activities, and sedentary behaviours: cross-sectional findings from a national cohort of over 70000 Thai adults. BMC Public Health 2011,11:762

21. Pedrinelli A, Garcez-Leme LE, Nobre RC. O efeito da atividade física no aparelho locomotor do idoso. Rev. Bras.Ortop. 2009; 44:96-101.

22. Sacco I C, Sartor CD, Gomes AA, João SMA, Cronfli R. Avaliação das perdas sensório-motoras do pé e tornozelo decorrentes da neuropatia diabética. Rev. Bras. Fisioter. 2007; 11: 27-33.

23. Huang PL. eNOS, metabolic syndrome and cardiovascular disease. Trends Endocrinol Metab 2009; 20:295-302.

24. Zaros PR, Pires CE, Bacci M Jr, Moraes C, Zanesco A. Effect of 6-months of physical exercise on the nitrate/nitrite levels in hypertensive postmenopausal women. BMC Womens Health. 2009;9:17.

25. Nagata D, Hirata Y. The role of AMP-activated protein kinase in the cardiovascular system. Hypertension Research 2010; 33:22-8.

26. Fernandes RA, Christofaro DG, Casonatto J, Codogno JS, Rodrigues E, Cardoso M, Kawaguti S, Zanesco A. Prevalence of dyslipidemia in individuals physically active during childhood, adolescence and adult age. Arq Bras Cardiol. 2011; 97:317-323. 